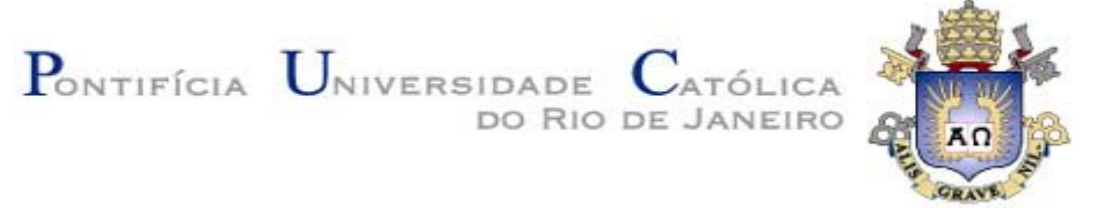

Martim Francisco de Oliveira e Silva

\author{
A Vantagem Competitiva das Nações \\ e a Vantagem Competitiva das Empresas:
}

A Localização é Importante?

Tese apresentada ao Programa de Pós-graduação em Administração de Empresas da PUC-Rio como requisito parcial para obtenção do título de Doutor em Administração de Empresas.

Orientador: Jorge Ferreira da Silva 


\title{
A Vantagem Competitiva das Nações e a Vantagem Competitiva das Empresas: \\ A Localização é Importante?
}

Tese apresentada como requisito parcial para obtenção do grau de Doutor pelo Programa de Pósgraduação em Administração de Empresas da PUCRio. Aprovada pela Comissão Examinadora abaixo assinada.

\author{
Prof. Jorge Ferreira da Silva \\ Orientador \\ Departamento de Administração - PUC-Rio \\ Prof. Luiz Felipe Jacques da Motta \\ Departamento de Administração - PUC-Rio \\ Profa. Angela Maria Cavalcanti da Rocha \\ COPPEAD/UFRJ
}

Prof. Agrícola de Souza Bethlem COPPEAD/UFRJ

Prof. Clóvis Luiz Machado-da-Silva UFPR 
Todos os direitos reservados. É proibida a reprodução total ou parcial do trabalho sem a autorização da universidade, do autor e do orientador.

\section{Martim Francisco de Oliveira e Silva}

Graduado em Engenharia Civil pela Universidade Federal do Rio de Janeiro em 1984. Mestre em Administração de Empresas pela Pontifícia Universidade Católica do Rio de Janeiro em 1991. Atuou por 27 anos nas indústrias química, farmacêutica, cosmética e de telecomunicações.

Ficha catalográfica

Silva, Martim Francisco de Oliveira e

A vantagem competitiva das nações e a vantagem competitiva das empresas : a localização e importante? / Martim Francisco de Oliveira e Silva ; orientador: Jorge Ferreira da Silva. - 2009.

204 f. : il. ; $30 \mathrm{~cm}$

Tese (Doutorado em Administração)-Pontifícia Universidade Católica do Rio de Janeiro, Rio de Janeiro, 2009.

Inclui bibliografia

1. Administração - Teses. 2. Efeito país. 3. Competitividade dos países. 4. Desempenho de empresas. 5. Vantagem competitiva das nações. I. Silva, Jorge Ferreira da. II. Pontifícia Universidade Católica do Rio de Janeiro. Departamento de Administração. III. Título. 


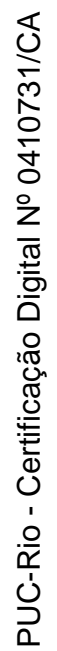

Ao meu pai. 


\section{Agradecimentos}

Ao meu orientador, Professor Jorge Ferreira da Silva, por todo o aconselhamento e por mais um incentivo a esta nova conquista acadêmica.

Ao Professor Luiz Felipe Jacques da Motta, pela participação na idéia inicial que originou esta pesquisa e por me proporcionar as condições para concluí-la.

Aos Professores Agrícola Bethlem e Angela da Rocha pelas contribuições durante o exame de qualificação para esta tese.

Para Ivan Marques, pelo acesso à base de dados do MSCI Barra.

Aos professores do Programa de Doutorado em Administração de Empresas da PUC - Rio, pelo incentivo e conhecimentos transmitidos.

Ao pessoal administrativo do Departamento de Administração, em especial, ao Fabio Etienne e a Teresa Campos.

À minha mãe, Maria Eunice, tanto pelo auxílio na revisão do texto final, como pela presença permanente.

Finalmente, um especial agradecimento à minha esposa, Daniela, pela paciência e compreensão diante de minhas ausências motivadas pelo desafio deste projeto acadêmico. 


\section{Resumo}

Silva, Martim Francisco de Oliveira e; Silva, Jorge Ferreria da. A Vantagem Competitiva das Nações e a Vantagem Competitiva das Empresas: A Localização é Importante?. Rio de Janeiro, 2009. 204p. Tese de Doutorado - Departamento de Administração, Pontifícia Universidade Católica do Rio de Janeiro.

Há dois pontos de vista dominantes no campo da Administração Estratégica para explicar o desempenho das empresas: a visão da Organização Industrial, que destaca o papel do ambiente industrial e a Visão Baseada em Recursos, que salienta a importância das características individuais das empresas, ambos extensamente estudados. Entretanto, a associação entre o desempenho das empresas e a competitividade das nações, em termos de seus ambientes institucionais, culturais, políticos e econômicos é um campo do conhecimento que continua a ser pouco explorado. Este estudo buscou comprovar que o desempenho das empresas varia não apenas em função das indústrias a que elas pertencem ou de suas características individuais, mas também em função do ambiente mais geral dos países em que elas operam. A partir dos relatórios anuais de competitividade do World Economic Forum e dos indicadores de desempenho obtidos a partir da base de dados do MSCI Inc., foram testadas as hipóteses formuladas associando o ambiente mais geral dos países ao desempenho de suas empresas, sendo encontradas evidências da existência do relacionamento entre os indicadores de competitividade dos países e o desempenho sustentável de suas empresas. $\mathrm{O}$ estudo tratou de maneira pioneira o relacionamento entre o conceito da vantagem competitiva das nações, através de seus indicadores de competitividade e a vantagem competitiva das empresas, por meio de seu desempenho; testou empiricamente o modelo do Diamante Competitivo do Professor Michael Porter; destacou três variáveis, habitualmente negligenciadas na linha de pesquisas das fontes de desempenho de empresas: a Sofisticação dos Compradores, o PIB e as Compras Governamentais; e criou um novo indicador de desempenho que também traduz a sua sustentabilidade, o qual se relaciona à linha da pesquisa da persistência dos retornos anormais. 


\section{Palavras-chave}

Efeito país; competitividade dos países; desempenho de empresas; vantagem competitiva das nações. 


\section{Abstract}

Silva, Martim Francisco de Oliveira e; Silva, Jorge Ferreria da (Advisor). The Competitive Advantage of Nations and the Competitive Advantage of Companies: Does Location Matter? Rio de Janeiro, 2009. 204p. Tese de Doutorado - Departamento de Administração, Pontifícia Universidade Católica do Rio de Janeiro.

There are two dominant points of view in the field of Strategic Management to explain the firm performance: the Industrial Organization view, which highlights the role of the industrial environment, and the Resource Based View, which emphasizes the importance of the idiosyncratic characteristics of firms, both extensively studied. However, the association between the firm performance and the competitiveness of nations in terms of their institutional, cultural, political and economical environment is a field of knowledge still insufficiently explored. This study shows that firm performance depends not only on the industries to which they belong or to their individual characteristics, but also to the wider environment of the countries where they operate. From the Annual Competitiveness Reports of the World Economic Forum and the financial performance indicators obtained from the database of MSCI Inc., the hypothesis made relating the wider environment of the countries to the performance of their firms were tested. Evidences of the relationship between the nation's competitiveness indicators and the sustainable performance of their firms were found. The study pioneered the approach relating the concept of competitive advantage of nations, through its indicators of competitiveness, to the competitive advantage of companies, through their performance; empirically tested the model of Michael Porter's Competitive Diamond; highlighted three variables, usually neglected in the line of research of the sources of firm performance: Buyers Sophistication, GDP and Government Purchases; and created a new indicator of performance that also reflects its sustainability, which relates to the line of research on the persistence of abnormal returns.

\section{Keywords}

Country effect; country competitiveness; firm performance; competitive advantage of nations. 


\section{Sumário}

1. Introdução 16

$\begin{array}{ll}\text { 1.1. Objetivos } & 19\end{array}$

1.2. Metodologia 20

1.3. Contribuições 20

1.4. Importância

2. Revisão da Literatura 23

2.1. Estratégia 23

2.2. A Organização Industrial 27

2.3. A Visão Baseada em Recursos 37

2.4. Os Estudos da Importância Relativa 47

2.4.1. A Inclusão do Efeito do País 55

2.5. A Competitividade das Nações 59

2.5.1. Os Rankings de Competitividade 83

2.6. Desempenho de Empresas 92

2.6.1. A sustentabilidade do Desempenho 104

3. Hipóteses Obtidas a Partir da Literatura 106

4. Procedimentos Metodológicos 110

4.1. Fontes de Dados 110

4.2. Procedimentos Quantitativos 113

4.2.1. Amostra 113

4.2.2. Procedimento Estatístico para Efetuar as Regressões 120

4.3. Limitações 139

5. Análises dos Resultados 143 
6. Conclusões 155

6.1. Sumário do Estudo 155

6.2. Contribuições 158

6.3. Novas pesquisas 160

7. Referências Bibliográficas 163

8. Anexos 191 


\section{Lista de figuras}

Figura 2.1 - Os quatro elementos da formulação estratégica Segundo Porter(1981)

Figura 2.2 - O paradigma industrial segundo Bain (1951) e Mason (1939)

Figura 2.3 - Os pilares da vantagem competitiva segundo Peteraf (1993)

Figura 2.4 - O relacionamento entre a tradicional análise de forças, fraquezas, oportunidades e ameaças, os modelos de VBR e da atratividade industrial segundo Barney(1991)

Figura 2.5 - O Modelo do Diamante Competitivo de Porter (1990) 69

Figura 2.6 - Esquema classificatório das medidas de Desempenho Econômico segundo Venkatraman e Ramanujam (1986)

Figura 3.1 - O relacionamento entre o desempenho das empresas e as características do ambiente dos países

Figura 4.1 - O modelo adotado na pesquisa 


\section{Lista de gráficos}

Gráfico 4.1 - Taxa de poupança nacional e PBV do País

Gráfico 4.2 - Custos do terrorismo para os negócios e PBV do País 126

Gráfico 4.3 - Impacto da tuberculose nos Negócios e PBV do País

Gráfico 4.4 - Qualidade do fornecimento de energia elétrica e PBV do País

Gráfico 4.5 - Sofisticação dos compradores e PBV/SD País

Gráfico 4.6 - PIB e PBV/SD País

Gráfico 4.7 - Compras governamentais de produtos com tecnologia avançada e PBVISD País 


\section{Lista de tabelas}

Tabela 2.1 - Resumo dos estudos descritos sobre a importância relativa

Tabela 2.2 - Decomposição das fontes do desempenho, segundo Brito e Vasconcelos (2005)

Tabela 2.3 - Número de países incluídos nos relatórios anuais de competitividade do WEF

Tabela 4.1 - Indústrias e número de países na base de dados do $\mathrm{MSCl}$

Tabela 4.2 - PBV, SD, PBV/SD, o número médio de indústrias e o número médio de empresas presentes nos dados mensais do $\mathrm{MSCl}$ no período de 2002 até 2006

Tabela 4.3 - Estatísticas Descritivas - PBV, SD, PBV/SD, o número médio de indústrias e o número médio de empresas presentes nos dados mensais do $\mathrm{MSCl}$ no período de 2002 até 2006

Tabela 4.4 - Testes de Normalidade com os indicadores de desempenho

Tabela 4.5 - Estatísticas descritivas de PBV para países desenvolvidos e emergentes

Tabela 4.6 - Teste de diferenças entre médias de PBV de países desenvolvidos e emergentes

Tabela 4.7 - Teste de Wilcoxon para diferença de médias do indicador PBVISD entre países emergentes e desenvolvidos 
Tabela 4.8 - Resultados da regressão múltipla pelo método stepwise, com o indicador de desempenho PBVISD

Tabela 4.9 - Coeficientes da regressão múltipla e valores do teste t com o uso do método stepwise, com o indicador de desempenho PBVISD

Tabela 4.10 - Matriz de correlações das três variáveis extraídas pelo método stepwise

Tabela 4.11 - Análise de resíduos - todos os países

Tabela 4.12 - Resultados da regressão múltipla pelo método stepwise, com a retirada dos Estados Unidos da amostra e usando o indicador de desempenho PBV/SD

Tabela 4.13 - Coeficientes da regressão múltipla e valores do teste t com o uso do método stepwise, com a retirada dos Estados Unidos da amostra e usando como indicador de desempenho PBVISD

Tabela 4.14 - Análise de resíduos - Todos os países excluindo os Estados Unidos 


\section{Tabelas do anexo}

Tabela A.1 - Indicadores de competitividade e suas descrições

192

Tabela A.2 - Resultados dos testes de normalidade

199

Tabela A.3 - Regressões Simples: Indicadores de Competitividade e PBV

Tabela A.4 - Regressões Simples: Indicadores de Competitividade e PBVISD

Tabela A.5 - Comparação de médias entre indicadores de competitividade dos países emergentes e desenvolvidos

Tabela A.6 - Lista de países presentes nos Relatórios Anuais de Competitividade do IMD e do WEF 